Methods The previous e-ELCA lead worked with a team to map e-ELCA against the Association for Palliative Medicine (APM) medical school curriculum, identifying 64 relevant sessions. Further work from a team at Liverpool Medical School identified sessions that covered areas of the APM curriculum that were not covered by their local medical school curriculum. This previous work was combined to generate a provisional medical student learning path. This was presented at the APM undergraduate education special interest forum (APMUESIF). Feedback was gained on the choice of sessions but also the size and structure of the learning path. This feedback and the provisional learning path were used alongside the General Medical Council's (GMC's) Outcomes for Graduates document and the APM curriculum to produce a final learning path.

Results A compact medical student learning path comprising 5 core, 5 additional and 5 case study sessions has been developed.

Conclusions An e-ELCA medical student learning path has been developed following guidance from a number of sources. This will allow e-ELCA to be used more readily for undergraduate medical education.

\section{EXPANDING THE PROVISION OF PALLIATIVE MEDICINE EDUCATION ON THE GRADUATE ENTRY MEDICINE COURSE AT SWANSEA UNIVERSITY}

Annell Prosser, Idris Baker, Hannah Walrond. Swansea University, Abertawe Bro Morgannwg University Health Board

\subsection{6/bmjspcare-2019-ASP.47}

Background The provision of Palliative Medicine education within UK Medical Schools is a subject of ongoing discussion, with wide variation in how much teaching is delivered. With $36 \%$ of hospital patients having palliative care needs, all newly qualified doctors require these core skills. At Swansea University, this teaching has previously been scattered throughout the curriculum with little co-ordination. We were given the opportunity to develop a new combined Pain and Palliative Medicine learning week for second year students, aiming to design a structured and balanced week to complement clinical teaching.

Methods Our working group included a third year medical student, who helped to focus our teaching techniques on what would be appropriate for her peers - mature and engaged adult learners with well-developed learning skills. We surveyed previous students to attain their thoughts on existing and future learning opportunities; with responses influential in the development of the week. A similar survey was sent to those who attended the week, to ensure their learning needs were met.

Results There was a 51\% absolute increase in those who felt they had sufficient teaching on Palliative Medicine after this week; with a $26 \%$ increase in those who felt confident or reasonably confident in their knowledge of Palliative Medicine, and $14 \%$ in their communication skills in Palliative Medicine. We will compare with APM data on medical education and consider next steps, including our current work, in using this year's feedback to shape next year's teaching.

Conclusions This exciting opportunity to expand the Palliative Medicine teaching at Swansea University is a further step towards building a workforce better equipped to deliver general palliative care, as well as nurturing and inspiring the next generation of experts. We will present learning from how the week was developed and thinking on how to evolve for the future.

\section{INTEGRATING HOSPICE AND END OF LIFE CARE INTO THE UNDERGRADUATE MEDICAL CURRICULUM: EVALUATION OF A NEW UNDERGRADUATE EDUCATIONAL PLACEMENT IN NORTH STAFFORDSHIRE}

Melica Daily-Hunt, Claire L Hookey. Douglas Macmillan Hospice, University Hospital of the North Midlands, Keele University

\subsection{6/bmjspcare-2019-ASP.48}

Background Although palliative care teaching for undergraduate students in the UK is increasing, there remains considerable variation between medical schools. It is unclear how much opportunity there is for experiential learning from patient contact (Walker, S et al. 2016). End of life care is integrated throughout Keele Medical School's spiral curriculum, but it has previously not been possible for all students to experience or even visit a hospice. A half-day placement at Douglas Macmillan Hospice, Stoke-on-Trent, during the third year elderly care attachment was piloted. Viability and student satisfaction were evaluated.

Method All third year medical students at Keele University attended a half-day placement at Douglas Macmillan Hospice in groups of up to six students. This included hospice orientation, observation of a nurse-doctor handover and syringe driver administration, and a patient interview in pairs followed by a de-brief. Students completed a feedback questionnaire including numerical rating scales and free text responses. Medical staff involved in organising the placement assessed the resource implications for the hospice in providing it.

Results 89 students attended. $81 \%$ completed a feedback questionnaire. $78 \%$ had never visited a hospice before. Numerical ratings for all aspects of the placement were high indicating that students valued the experience. The most frequent suggestion for improvement was a longer placement.

Conclusions The placement was highly valued by students but challenging to deliver due to the time needed from staff and the large number of students. The positive student feedback enabled funding to be secured to support the hospice to continue to provide the placement. Additionally, this academic year a palliative care seminar within the same elderly care attachment will explore end of life care in dementia. Further evaluation is needed to establish the effectiveness of the placement in influencing students' learning and behaviour in end of life care.

\section{REDESIGNING THE REGIONAL TEACHING PROGRAM FOR PALLIATIVE MEDICINE}

Katherine Stewart, Joanna Roberts, Grace Ting, Rebecca Singh-Curry, Clare Forshaw, Jennifer Wiseman, Esraa Sulaivany, Michelle Tombs, Andrew Fletcher, Andrew Khodabukus. St Rocco's Hospice

\subsection{6/bmjspcare-2019-ASP.49}

Background Traditionally the trainees in the North West and Mersey deaneries have run separate teaching programs. After NW and Mersey deaneries merged the decision was 
made to have one education program and provide a regional day of teaching on alternate months. Attendance is mandatory for trainees, but open to all doctors working in the specialty.

We designed a 4 year program of topics based on the specialty curriculum. Each half day covers one topic and is organised by a trainee from either NW or Mersey with consultant support. The program is overseen by $\mathrm{SpR}$ leads who manage the email account, registration for each day and support for session organisers. Trainees organising each half day are encouraged to lead a session, enabling them to get formal feedback and a teaching observation completed.

Methods We ran this program for 12 months before completing a review. 2 surveys were sent out using survey monkey. One was sent to all attendees and another to those who had organised sessions. The demographics of attendees were also analysed.

Results Sessions have been attended by a mix of trainees, consultants and SAS doctors, with 30-35 attendees per day. $84 \%$ of attendees said the sessions met their learning needs either well or very well. $92 \%$ rated the sessions as good or excellent. 92\% of session organisers felt they had enough support and information. The main challenge was finding speakers, which $58 \%$ reported to be difficult or very difficult.

Conclusions Feedback has led to the creation of a checklist to support those organising sessions. Senior SAS doctors will be more involved, making the most of expertise in the region. The new program has been well received. It provides education that meets the needs of the training program whilst supporting other doctors working in the specialty.

\section{\begin{tabular}{|l|l}
\hline 27 & IMPROVING END OF LIFE CARE IN NURSING HOMES
\end{tabular}}

Magdalen Thomson, Stephanie Killick, Sarah McGaughey, Tracey Platt, Rachel Gaffney. Hampshire Hospitals NHS Foundation Trust, Brighterkind Care Homes, West Hampshire Clinical Commissioning Group

\subsection{6/bmjspcare-2019-ASP.50}

Background There were 716 deaths in Royal Hampshire County Hospital and Andover Ward Memorial hospitals (including hospice) in 2016. With no hospice beds in Winchester $25 \%$ patients die in a care home opposed to $18 \%$ nationally. The team felt that the quality of care homes was variable, staff lack confidence and competence, symptom were not always well controlled, and lack of advanced care planning resulted in inappropriate hospital admissions.

Method One year quality improvement project carried out by a cross -organisational team including a commissioner, a community pharmacist, two care home managers, a specialist palliative care team Consultant, and two palliative care clinical matrons from acute hospital and hospice. The project was supported by NHS Wessex. The PDSA cycle used mapping of existing discharge processes, stakeholder analysis, a driver diagram, and a risk chart. Quantitative and qualitative interview data was collected. Changes were implemented at regular intervals throughout the year and education was provided. Initial syringe driver usage was also measured.

Quantitative results Each care home rating out of 10 rose from 7.6 and 8.6, to 9.5 and 9.6 respectively. One received an 'outstanding' CQC rating for end of life care. 3 core qualitative questions were asked to measure confidence and competence. At the start of the project $67 \%$ responded positively, which rose to $100 \%$ by the end. Staff and family feedback was overwhelmingly positive and there were no inappropriate hospital admissions. There were several unintended benefits including better inter-rational working for between hospital and care homes, increased levels of trust, better quality of information, and staff felt empowered to influence care provided by GPs.

Conclusion The study showed that this had been a successful project with several additional unintended benefits. The team are planning to replicate the project in further care homes and fund raising is well underway to open Winchester hospice next year (2019).

\section{THE USE OF SUBCUTANEOUS FUROSEMIDE FOR THE SYMPTOMATIC MANAGEMENT OF PATIENTS WITH END STAGE HEART FAILURE IN THE COMMUNITY; A GP SURVEY}

Emma Lundy. Belfast Health and Social Care Trust, Northern Health and Social Care Trust, Western Health and Social Care Trust, Southern Health and Social Care Trust, South Eastern Health and Social Care Trust

\subsection{6/bmjspcare-2019-ASP.51}

Heart failure (HF) is appropriately described as an epidemic with recent estimates suggesting there are more than half a million people living with this syndrome in the UK alone.

For patients with end-stage HF (ESHF), diuretic therapy is the cornerstone of treatment. These patients frequently require escalation to parenteral diuretics for symptom management; this can be a challenging option for patients who wish to be cared for at home. Continuous subcutaneous infusion (CSCI) of medication via a syringe driver (SD) has been used for over 30 years, revolutionising the care of dying people. Administering furosemide via this route can be an effective alternative to intravenous (IV) therapy.

An electronic survey was sent to General Practitioners (GPs) across the five Health and Social Care Trusts in Northern Ireland (NI) to determine if subcutaneous furosemide was being prescribed for patients in the community with ESHF. The survey was disseminated via email and private social media sites.

63 GPs completed the survey. $86 \%$ of GPs did not know that furosemide could be given subcutaneously with only $5 \%$ of GPs having ever prescribed it. 90\% of GPs felt that there was a role for subcutaneous furosemide and under specialist guidance $86 \%$ of GPs would be willing to prescribe it. 44\% of GPs felt that they would need additional training to feel confident in prescribing subcutaneous furosemide and expressed concerns, such as, availability of medication, training for district nurses, additional GP workload, anxiety relating to blood monitoring and choosing the appropriate dose.

Subcutaneous furosemide can be beneficial in the symptomatic management of patients with ESHF. With specialist guidance, training and support GPs in NI would be willing to consider this as a treatment option, which could prevent unnecessary hospital admissions and allow patients with ESHF to die comfortably in their own home. 